University of Nebraska - Lincoln

DigitalCommons@University of Nebraska - Lincoln

November 1993

\title{
Monte Carlo simulation of the solid to super liquid phase transition of Langmuir monolayers using cross-section potentials
}

\author{
D.R. Swanson \\ University of Nebraska - Lincoln \\ Robert J. Hardy \\ University of Nebraska - Lincoln, rhardy1@unl.edu \\ Craig J. Eckhardt \\ University of Nebraska - Lincoln, ceckhardt1@unl.edu
}

Follow this and additional works at: https://digitalcommons.unl.edu/chemistryeckhardt

Part of the Chemistry Commons

Swanson, D.R.; Hardy, Robert J.; and Eckhardt, Craig J., "Monte Carlo simulation of the solid to super liquid phase transition of Langmuir monolayers using cross-section potentials" (1993). Craig J. Eckhardt Publications. 14.

https://digitalcommons.unl.edu/chemistryeckhardt/14

This Article is brought to you for free and open access by the Published Research - Department of Chemistry at DigitalCommons@University of Nebraska - Lincoln. It has been accepted for inclusion in Craig J. Eckhardt Publications by an authorized administrator of DigitalCommons@University of Nebraska - Lincoln. 


\title{
Monte Carlo simulation of the solid to super liquid phase transition of Langmuir monolayers using cross-section potentials
}

\author{
D. R. Swanson \\ Department of Chemistry, University of Nebraska-Lincoln, Lincoln, Nebraska 68588
}

R. J. Hardy

Department of Physics, University of Nebraska-Lincoln, Lincoln, Nebraska 68588

C. J. Eckhardt ${ }^{\text {a) }}$

Department of Chemistry and Department of Physics, University of Nebraska-Lincoln, Lincoln, Nebraska 68588

(Received 29 June 1993; accepted 2 August 1993)

\begin{abstract}
A cross-section potential which models the interaction of two planar objects of prescribed shape is developed and used to model the interactions of amphiphiles in vertical phases of Langmuir monolayers. The results of Monte Carlo simulations of an isobaric-isothermal ensemble show qualitative agreement with experiment. The model system undergoes a continuous, bound-to-free rotator transition between phases of the same symmetries as the solid (S) and super liquid (LS) phases exhibited by fatty acid films.
\end{abstract}

\section{INTRODUCTION}

Phase transitions in Langmuir monolayers, films formed by spreading molecules with both hydrophilic and hydrophobic regions on an aqueous surface, are relevant to research ranging from molecular switches exploitable for nanotechnology to lipid membranes fundamental for sustaining life. ${ }^{1}$ The current status of the subject has been reviewed elsewhere. ${ }^{2-4}$ The component molecules of such a film, commonly referred to as amphiphiles, may be ordered conformationally, orientationally, and translationally by applying a surface pressure $\pi$ at a sufficiently low temperature $T$. Although much detail concerning these systems' $\pi, T$ phase diagrams has now been determined experimentally, 5,6 the mechanisms of the various phase transitions examined remain unclear due, in part, to a lack of successful simple models. This paper presents a simple model for the transition between two of the untilted phases.

The preponderance of experimental, theoretical, and computational work published on Langmuir monolayers has been performed on amphiphiles composed of fatty acids or their derivatives. Langmuir originally proposed that the intermolecular packing of these monolayers is determined primarily by the hydrophobic tails of the amphiphiles. ${ }^{7}$ Lundquist ${ }^{8}$ showed that monolayer solid phases form by employing arguments based upon the chirality of amphiphiles with structures similar to those of fatty acids. Recently, Eckhardt et al. utilized chirality arguments ${ }^{9}$ in interpreting results of experiments performed on films composed of chiral rigidified amphiphiles with chemical structures radically different from that of fatty acids. Atomic force microscopy images of transferred films confirm that the chirality of the amphiphile significantly constrains the possible symmetries of the resulting lattice.

These results indicate that the intermolecular packing exhibited by such films is dictated primarily by the cross

\footnotetext{
a) Author to whom correspondence should be addressed.
}

section of the constituent amphiphile. For amphiphiles with a regular shape, this model gives a first-order indicator of the expected film packing. The resulting twodimensional space groups of several systems have been explained utilizing simple geometric arguments, coupled with straightforward atom-atom potential calculations, that assume a rigid, vertical molecule. ${ }^{10,11}$ These assumptions are reasonable for condensed phases at sufficient pressure. If one additionally constrains the head groups of such a film to a plane, it is clear that any planar slice parallel to the film plane contains uniform cross sections. This will not be true if the molecular conformation or tilt is not constant for the phase under consideration. This paper investigates these arguments by creating a particularly simple model. If the cross section of the amphiphile indeed determines packing, a two-dimensional simulation of particles with appropriate cross section should give results analogous to those found for Langmuir monolayers.

Though there has been some discussion of the character and universality of fatty acid monolayer phases, ${ }^{4,12}$ the untilted phases, which have the long axis of the amphiphiles parallel to the film normal, are consistently observed. ${ }^{6,13,14}$ These high-pressure phases are often referred to as the super liquid (LS), solid (S), and crystalline solid (CS) phases. ${ }^{15}$ They are analogous to several of the phases found in three-dimensional paraffin crystals. ${ }^{16-18}$ The LS phase has hexagonal symmetry and liquid-like viscosity. It is analogous to the free rotator phase (rotator II) that exists at higher temperatures in paraffins of sufficient length. The $S$ and $C S$ phases both pack in a centered rectangular net, but the $S$ phase has more orientational disorder and slightly different intermolecular spacings. Its average structure, also centered rectangular, is analogous to that of the rotator I phase in paraffin crystals in which molecular rotations are restricted. It is the $S$ to LS transition that is modeled here.

An early two-dimensional molecular dynamics simulation applied to fatty acid monolayers was carried out by 
Cotterill. ${ }^{19}$ His model was composed of diatomic molecules and exhibited an order-disorder phase transition. Toxvaerd ${ }^{20}$ derived an equation of state for a two-dimensional fluid, but was limited in the evaluation of his results by the lack of experimental data at the time. A simple model that has recently received considerable attention is the system of rigid rods. ${ }^{21}$ It has had considerable success, but it does not reproduce all of the features of the observed phase diagrams. Specifically, it does not give transitions between different untilted phases because of the assumption of a circular cross section for the rods.

It is now almost routine to allow for all intra- and intermolecular degrees of freedom and to have the atoms interact through realistic and complex potential energy functions. ${ }^{22}$ However, we agree with the observation of Knobler and Desai ${ }^{4}$ that "theoretical progress will be linked more likely to simulations on model systems that are made progressively more complex as the results of simpler models are analyzed and understood theoretically." In the spirit of this, we consider the following simple model:

$N$ two-dimensional objects, or molecules, are allowed to rotate and translate freely in a plane under the influence of an anisotropic pair potential and an applied external pressure. The pair potential is chosen to approximate the interaction of two rigid objects with the cross section of an amphiphile molecule projected onto the plane of the film. We assume that the forces holding the amphiphiles parallel to the film normal effectively constrain each molecule to rotate as a rigid object. The translational freedom allows the system to take on different space group symmetries. At low temperatures, one expects such objects to be locked into a structure that is determined by the anisotropy of their profiles, while at temperatures for which $k T$ is greater than the energy needed to rotate an object, one expects the objects to rotate freely and the system to take on the closepacked hexagonal structure. These expectations are borne out by our simulations.

A Monte Carlo simulation of the isobaric-isothermal ensemble was used to investigate the implications of the model. Evidence was found for a continuous phase transition from a lower temperature, centered rectangular structure to a higher temperature, hexagonal structure. The orientations of the objects were found to be correlated in the lower temperature phase, but not in the higher temperature one. Shih et al. have used synchrotron $\mathrm{x}$-ray methods to study several films along isobars that intersect the three high pressure phases. ${ }^{13,14}$ The results of the simulations are in qualitative agreement with this high quality data.

\section{CROSS-SECTION POTENTIAL MODEL}

Our hypothesis is that the cause of phase transitions between different untilted phases in Langmuir monolayers is the anisotropy of the cross sections of the molecules. To model this, we consider a collection of $N$ two-dimensional anisotropic objects that are free to rotate and translate in a two-dimensional region which can vary in size and shape. The profile of the objects is chosen to approximate the cross section of an amphiphile molecule. An excluded volume plot of a paraffin molecule projected onto the plane

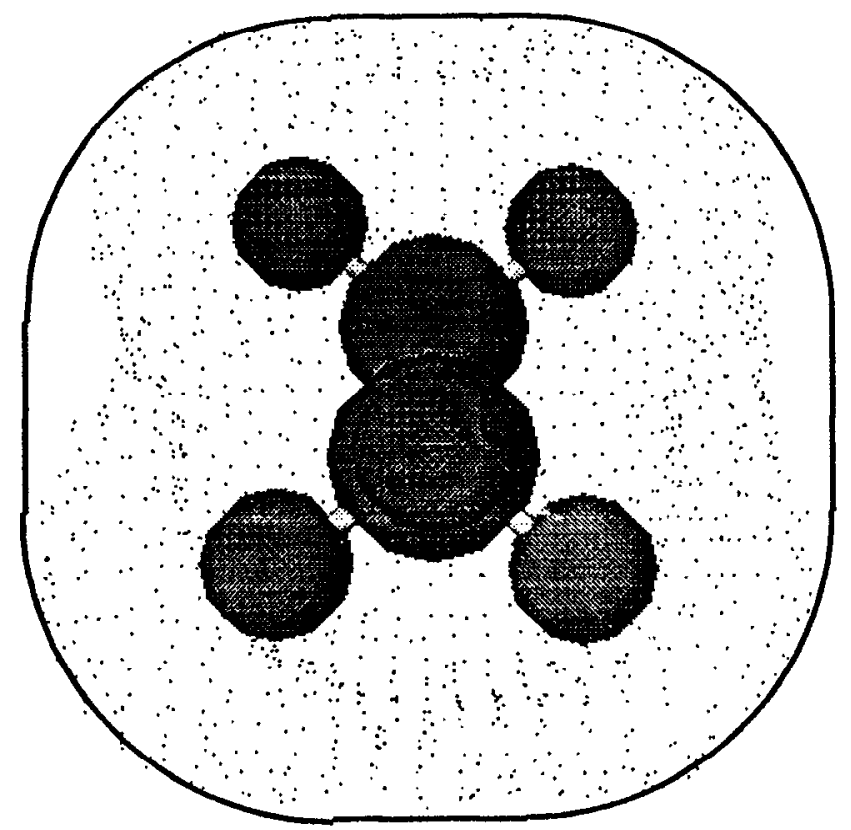

FIG. 1. A fatty acid cross section surrounded by an idealized perimeter representing the equation $\rho(\gamma)=\rho_{0}[1+\alpha \cos (4 \gamma)]$, where $\alpha=1 / 30$.

perpendicular to its long axis is shown in Fig. 1. The projection has the shape of a rounded rectangle. Since the length is only slightly different from the width, and since the emphasis here is on simplicity, objects with fourfold symmetry were considered. Extension to other crosssectional shapes is, however, straightforward.

The profile of a plane figure can be specified by a function $\rho(\gamma)$ that gives the dependence of $\rho$, the radial distance from the center, on $\gamma$, the angle from the direction of the $x$ axis. If the profile is close to circular, it is convenient to let

$$
\rho(\gamma)=\rho_{0}[1+\alpha g(\gamma)],
$$

where $\rho_{0}$ is the nominal radius of the object and where $\alpha$ and $g(\gamma)$ determine the magnitude and the type of the deviation from a circle, respectively. The profile given by the solid line in Fig. 1 was obtained with $\alpha=1 / 30$ and

$$
g(\gamma)=\cos 4 \gamma,
$$

which has fourfold symmetry.

Figure 2 shows the profiles of two molecules with the squarish shape described by Eqs. (1) and (2) and $\alpha=1 /$ 30. The perimeters of the molecules are in contact in Fig. 2, and the point of contact between them is on the straight line between their centers. This will not, in general, be the case. However, the displacement of the point of contact from the line between the centers will in general be small if $\alpha$ is small. For efficiency, the potential is evaluated in the limit where intermolecular contact is on the line between molecular centers. This limits the amount of anisotropy that can effectively be modeled, since highly anisotropic molecules will often collide in a nonlinear arrangement of the contact point and molecular centers. Further iterations 


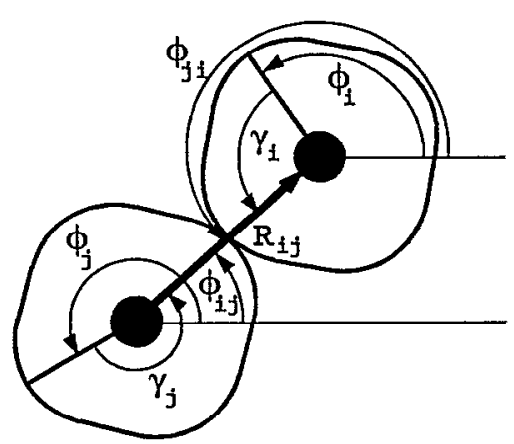

FIG. 2. Model system coordinates.

to account for such a nonlinear arrangement are possible, but were not performed to be consistent with the extreme simplicity of the model.

The angles $\phi_{i}$ and $\phi_{j}$ in Fig. 2 give the orientation of reference directions fixed in each of the molecules relative to a direction fixed in space, namely, the direction of the $x$ axis. $\gamma_{i}$ and $\gamma_{j}$ are the angles between these reference directions and a line drawn to the point of contact. The angle $\phi_{i j}$ gives the orientation of the vector between the centers of molecules $i$ and $j$ and is defined by

$$
\cos \phi_{i j}=\mathbf{r}_{i j} \cdot \hat{x} / r_{i j} \text {, }
$$

where $\mathbf{r}_{i j}=\mathbf{r}_{i}-\mathbf{r}_{j}$. Here, $r_{i j}$ is the magnitude of $\mathbf{r}_{i j}$, and $\hat{x}$ is a unit vector in the $x$ direction. It follows that $\phi_{j i}=\pi$ $+\phi_{i j}$, and from the figure, it may be seen that

$$
\phi_{i}+\gamma_{i}+\phi_{i j}=2 \pi \text { and } \phi_{j}+\gamma_{j}+\phi_{j i}=2 \pi \text {. }
$$

By using the fact that angles are only significant up to an additive constant of $2 \pi$ times an integer, these relations can be rearranged as

$$
\gamma_{i}=\phi_{i j}-\phi_{i} \text { and } \gamma_{j}=\phi_{j i}-\phi_{j} \text {, }
$$

which, with Eq. (3), gives the dependence of the angles $\gamma_{i}$ and $\gamma_{j}$ on the canonical variables, which are $x_{i}, y_{i}$, and $\phi_{i}$, where $\mathbf{r}_{i}=\left(x_{i}, y_{i}\right)$ and $i=1,2, \ldots, N$.

A modified Lennard-Jones potential is used. The method used to incorporate anisotropy is similar to, but simpler than, the method used by Kihara. ${ }^{23}$ The LennardJones pair potential has the form

$$
\phi(r)=\frac{A}{r^{12}}-\frac{B}{r^{6}},
$$

where $A$ and $B$ are constants. We take $r$ to be the distance between the centers of the molecules and associate the touching of the perimeters with the separation $r=r_{\mathrm{pm}}$ that minimizes the potential. It follows from Eq. (6) that the potential minimum $(\mathrm{pm})$ separation is

$$
r_{\mathrm{pm}}=(2 A / B)^{1 / 6} \text {. }
$$

The radii of the two molecules are assumed to be additive

$$
r_{\mathrm{pm}}=\rho\left(\gamma_{i}\right)+\rho\left(\gamma_{j}\right)
$$

and the attractive $-B / r^{6}$ term is assumed isotropic, so that all of the anisotropy appears in the repulsive $A / r^{12}$ term. These ideas are implemented by setting

$$
A=\left(2 \rho_{0}\right)^{6}\left[\rho\left(\gamma_{i}\right)+\rho\left(\gamma_{j}\right)\right]^{6} \epsilon \text { and } B=2\left(2 \rho_{0}\right)^{6} \epsilon \text {. }
$$

Substituting these into Eq. (6) gives an intermolecular potential that depends on the relative positions of the centers of the molecules and on their orientations

$\phi\left(r_{i j}, \gamma_{i}, \gamma_{j}\right)=\epsilon\left[\left(\frac{2 \rho_{0}}{r_{i j}}\right)^{12}\left[\frac{\rho\left(\gamma_{i}\right)+\rho\left(\gamma_{j}\right)}{2 \rho_{0}}\right]^{6}-2\left(\frac{2 \rho_{0}}{r_{i j}}\right)^{6}\right]$.

By using Eqs. (1) and (2) for $\rho(\gamma)$, expanding in powers of $\alpha$, neglecting higher order terms, and using Eq. (5) terms, one obtains

$$
\begin{aligned}
\phi\left(r_{i j}, \phi_{i j}, \phi_{i}, \phi_{j}\right)= & \epsilon\left(( \frac { 2 \rho _ { 0 } } { r _ { i j } } ) ^ { 1 2 } \left\{1+3 \alpha\left[\cos 4\left(\phi_{i j}-\phi_{i}\right)\right.\right.\right. \\
& \left.\left.\left.+\cos 4\left(\phi_{j i}-\phi_{j}\right)\right]\right\}-2\left(\frac{2 \rho_{0}}{r_{i j}}\right)^{6}\right),
\end{aligned}
$$

where $\phi_{i j}=\pi+\phi_{j i}$ is defined by Eq. (3). This is the crosssection potential that was used. It was smoothly truncated to zero at $\left|\mathbf{r}_{i j}\right|=2.245 \rho_{0}$. The interpretation of $\phi\left(\mathbf{r}_{i j}, \phi_{i j} \phi_{i}, \phi_{j}\right)$ in terms of the touching of the perimeters of rigid objects is only accurate when $\alpha$ is small. However, even when $\alpha$ is not small, Eq. (11) gives an anisotropic pair potential that can be used for studying the behavior of the untilted phases of monolayers. The value of $\alpha$ can be varied to investigate different experimental situations with larger values of $\alpha$ corresponding to greater anisotropy. When $\alpha=0$, there is no angular anisotropy and the potential reduces to the Lennard-Jones form. If $\alpha=1 / 6$, the repulsive term vanishes when $\phi_{i j}-\phi_{i}=\phi_{j i}-\phi_{j}=\pi / 4$, so that the potential has become unphysical.

Figure 3 shows the potential $\phi\left(r_{i j}, \phi_{i j}, \phi_{i}, \phi_{j}\right)$ as a function of $r_{i j}$ and the angle $\phi_{i}$ when $\phi_{j}$ is set to $\pi / 4$ and the direction of $\mathbf{r}_{i j}$ is parallel to the $x$ axis so that $\phi_{i j}=0$. The first cosine term in Eq. (11) is then replaced by $\cos 4\left(-\phi_{i}\right)$ and the second cosine term is replaced by -1 . For this direction of $\mathbf{r}_{i j}$, the global minima of the potential occur when $\phi_{i}, \phi_{j}=\pi / 4,3 \pi / 4,5 \pi / 4$, and $7 \pi / 4$. The energy difference between these minima and the intervening saddle points is $\Delta \phi=7.5 \alpha \epsilon$.

The potential energy function used in the Monte Carlo simulation was

$$
E=\sum_{0<i<j<N} \phi\left(r_{i j}, \phi_{i j}, \phi_{i}, \phi_{j}\right)+\pi A_{x} B_{y},
$$

where the sum is over all pairs and the potential is given by Eq. (11). $\pi$ is the external surface pressure and $A_{x} B_{y}$ is the area to which the $N$ molecules are confined. By adding the kinetic energy to $E$, one obtains the microscopic enthalpy. Canonical averages based on the isobaric-isothermal ensemble $^{24,25}$ were approximated by using the Metropolis algorithm and by determining the energy difference in the Boltzmann's factor $\exp (-\Delta E / k T)$ with the above expression for $E$.

As diagrammed in Fig. 4(b) the direction of $A$ was chosen as the direction of the $x$ axis, so that $A_{x}$ and $B_{y}$ are the $x$ and $y$ dimensions of the parallelogram within which the molecules were confined. Periodic boundary conditions 


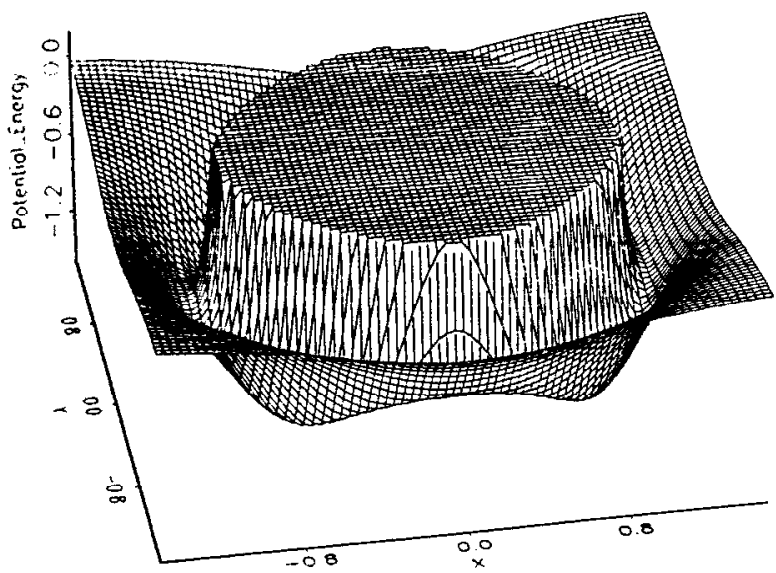

a

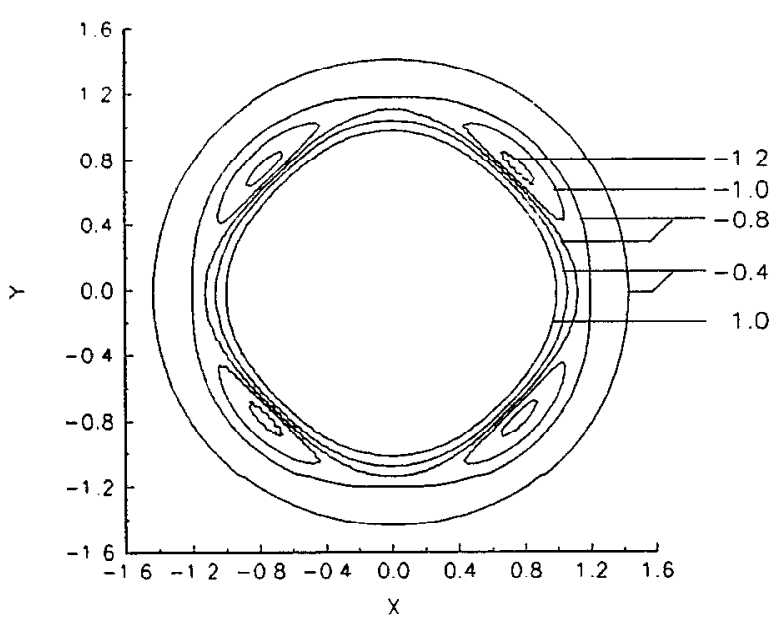

b

FIG. 3. (a) Reduced potential energy surface plotted as a function of $r_{i j}$ and $\phi_{i} . \phi_{j}=\pi / 4$ and $\phi_{i j}=0$. The hard core of the potential is cropped for clarity. (b) A contour plot of the potential energy surface. The contour lines along the hard core resemble the shapes drawn in Figs. 1 and 2.

were used, so that the point $\mathbf{r}$ and the points $\mathbf{r} \pm \mathbf{A} \pm \mathbf{B}$ are equivalent. Energy changes $\Delta E$ were calculated for random changes of the $3 N$ canonical variables $x_{i}, y_{i}$, and $\phi_{i}$ and for random changes of $A_{x}, B_{y}$, and $B_{x}$.

\section{CALCULATIONS AND RESULTS}

Figure 4(a) is a typical snapshot of the system at high temperature and shows molecules to be oriented randomly in a hexagonal arrangement. Figure $4(\mathrm{~b})$ is a snapshot at low temperature where the molecules are all oriented in approximately the same direction and the structure is centered rectangular.

The principal canonical averages calculated were the ratio $\langle b / a\rangle$ of the nearest to the next-nearest neighbor distances and its variance $\left\langle[(b / a)-\langle b / a\rangle]^{2}\right\rangle$. Distances $a$ and $b$ are indicated in Fig. $4(\mathrm{~b})$. In the hexagonal structure, $b / a=\sqrt{3}$. The lattice was found to always have one molecule per unit cell. For an array of $N_{\mathrm{A}}$ molecules in the A direction by $N_{\mathrm{B}}$ molecules in the $\mathrm{B}$ direction, one has
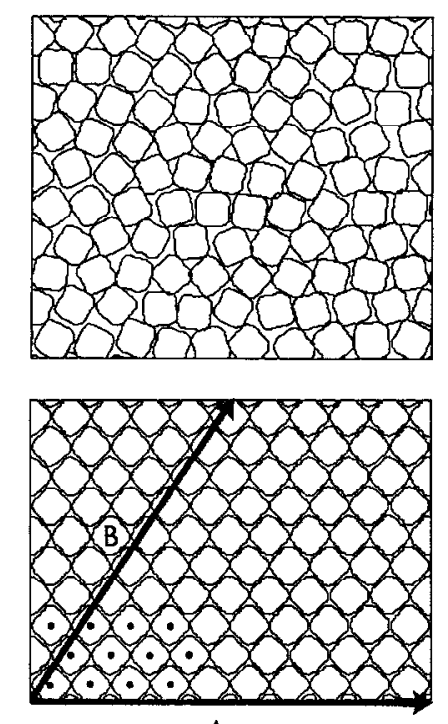

A

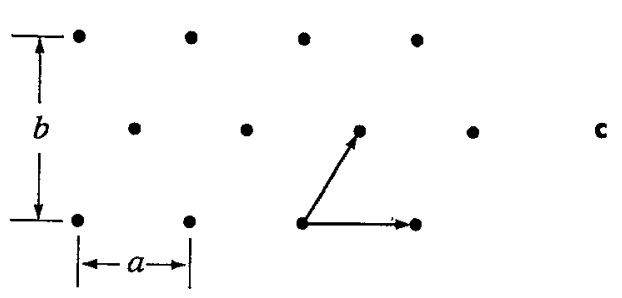

FIG. 4. (a) A snapshot of the high temperature $(T=0.200)$ phase. (b) A snapshot of the low temperature $(T=0.001)$ phase. $A$ and $B$ indicate vectors used to generate the periodic space. (c) A diagram illustrating $b$ and $a$, which for the systems studied, correspond to next-nearest and nearest neighbor distances, respectively. Average primitive lattice vectors, shown as bold arrows, may be derived directly from the periodic space vectors $A$ and $B$.

$a=A_{x} / N_{\mathrm{A}}$ and $b=2 B_{y} / N_{\mathrm{B}}$ [see Fig. 4(c)]. The variance is a generalized susceptibility. We also calculated the constant-pressure specific heat

$$
c_{\pi}=\left\langle[E-\langle E\rangle]^{2}\right\rangle /\left(N k T^{2}\right)+\frac{3}{2} k,
$$

where the second term contains momentum contributions.

To test the hypothesis that the molecules are free to rotate in the higher, but not in the lower temperature phase, we calculated the angular correlation coefficients defined by

$$
C^{g}=\frac{1}{N_{g}} \sum_{g}\left\langle\cos 4\left(\phi_{i}-\phi_{j}\right)\right\rangle
$$

where the number 4 is included to account for the fourfold symmetry of the molecules. The sum in Eq. (14) is over all pairs of molecules $i$ and $j$ within a particular group and $N_{g}$ is the number of pairs in the group. Two groups were considered. The first group with $g=1$ contains all pairs such that $1.78 \rho_{0}<r_{i j} \leqslant 2.85 \rho_{0}$. In the hexagonal phase, these are the nearest neighbor pairs. The second group with $g=2$ contains all pairs such that $2.85 \rho_{0}<r_{i j}<4.63 \rho_{0}$. In the hexagonal phase, these are the next-nearest neighbor pairs. No pairs were found in the immediate vicinity of $1.78,2.85$, or $4.63 \rho_{0}$. When the orientations of the molecules are ordered [Fig. 4(b)] the angles $\phi_{i}$ are all approx- 

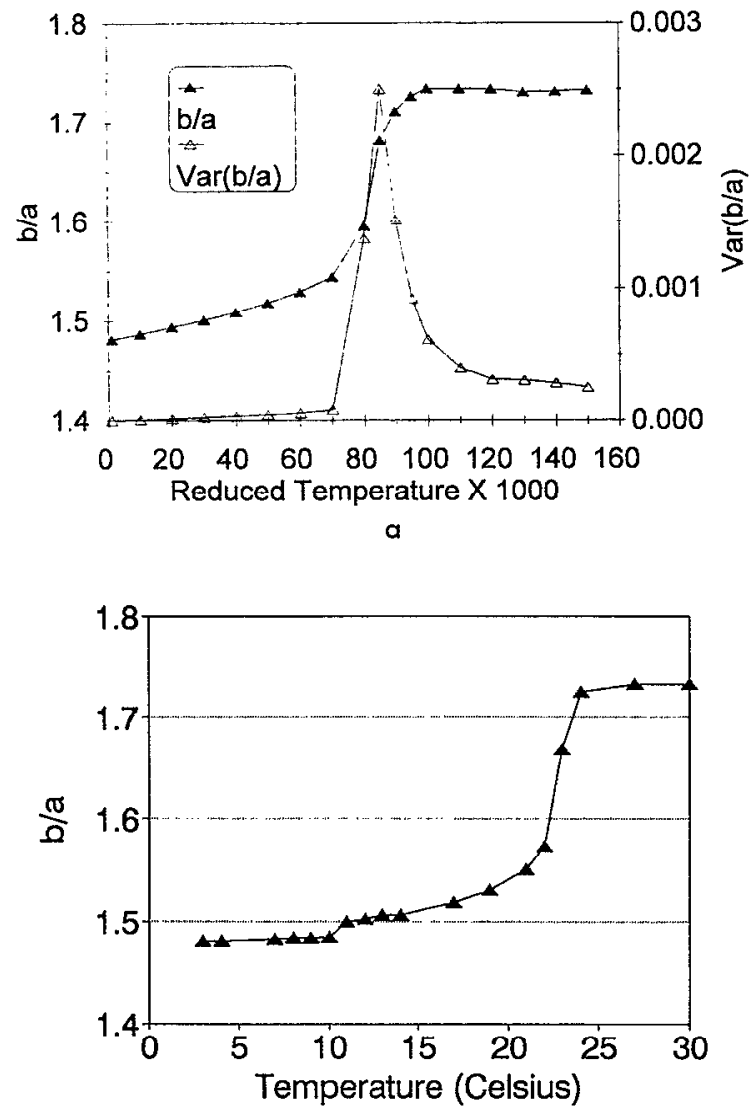

b

FIG. 5. (a) Simulated values of $\langle b / a\rangle$ and its variance as a function of reduced temperature. (b) Experimental values of $b / a$ as a function of temperature from Shih et al. (Ref. 13).

imately the same, so that $\phi_{i}-\phi_{j} \approx 0$ and $C^{g} \approx 1$. When the orientations are random [Fig. 4(a)], the angles $\phi_{i}$ and the differences $\phi_{i}-\phi_{j}$ take on all values between 0 and $2 \pi$ and $C^{g} \approx 0$.

The results given in Figs. 5, 6, and 7 are for $\alpha=1 / 30$, $\pi=0$, and $N=100$. Other values of $\alpha$ and $\pi$ were investigated, but no qualitatively different behavior was found. The transition temperature increases only slightly when either $\alpha$ or $\pi$ is increased. This is consistent with the sim-

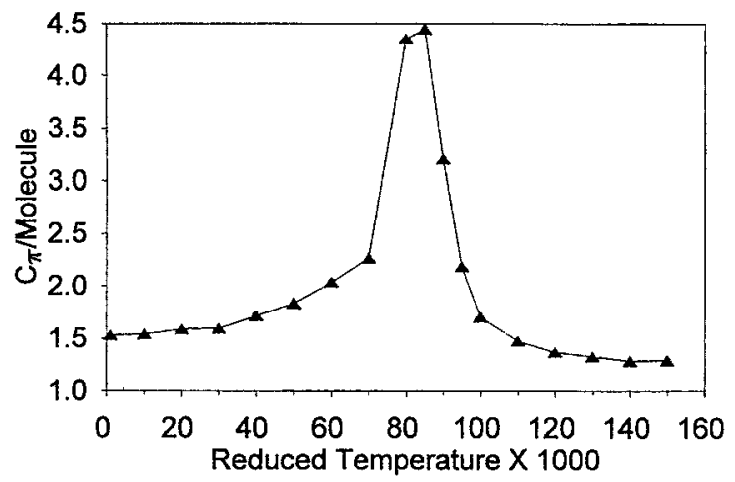

FIG. 6. Simulated values of $c_{\pi}$ as a function of reduced temperature (momentum contributions subtracted).

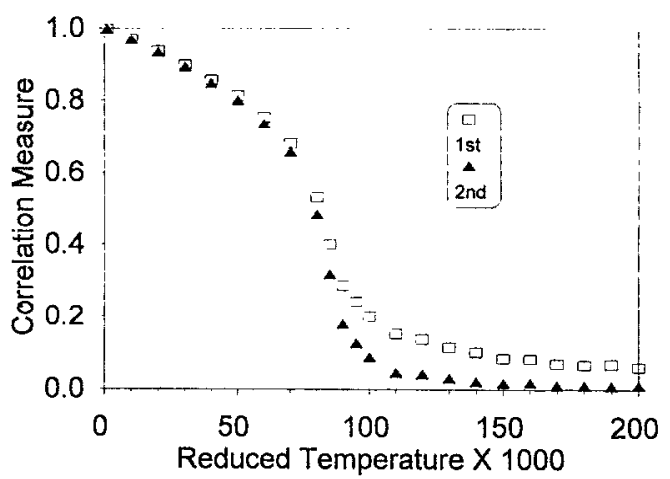

FIG. 7. Correlation measure $C^{8}$ as a function of reduced temperature for two groups of neighbors. The first group, which is the nearest neighbors for the hexagonal phase, includes all molecules found between 1.0 and 1.6 molecular diameters. The second group, which is the next nearest neighbors for the hexagonal phase, includes all molecules found between 1.7 and 2.6 molecular diameters. These groups contain the same molecules throughout the temperature range plotted.

plification that evaluates anisotropy only along the line between molecule centers. The initial $b / a$ value decreases with increasing $\alpha$, but increases slightly with increasing $\pi$. This mirrors the fact that the potential is more anisotropic near the minima than in the hard core region. Each data point represents an average over at least $10^{5}$ attempted moves per particle. After every $N$ attempted particle moves, one boundary move was attempted. The estimated statistical error in the data points is less than the size of the symbols used to plot them in the figures.

To verify that the model phase transition was not dictated by the periodic boundaries used, $N$ was varied from 64 to 400 and no significant differences were found. Furthermore, an attempted boundary move consists of making random changes in $A_{x}, B_{y}$, and $B_{x}$ and rescaling the positions of the centers of all the molecules without rotating them. This ensures that rectangular symmetry is not predetermined by the periodic space.

For comparison, the experimental results of Shih et al. ${ }^{13}$ for the ratio $\langle b / a\rangle$ are plotted in Fig. 5(b). Though this simple model does not scale to experimental values, the qualitative shape of this curve is obtained.

\section{DISCUSSION AND CONCLUSIONS}

The qualitative agreement between the experimental and Monte Carlo results in Fig. 5 suggests that the essential mechanism causing the transition between the $S$ and LS untilted phases in Langmuir monolayers is the anisotropy of the cross sections of the molecules. The simulation also suggests that the phase transitions are probably continuous, although the detailed study of size dependence needed to verify this has not been carried out. Nevertheless, no hysteresis was observed at the transition and both the variance and the specific heat diverge in the characteristic way.

The results for the correlation coefficients $C^{g}$ in Fig. 7 indicate that the orientations of the molecules are strongly correlated in the low temperature phase and rapidly be- 
come uncorrelated as the temperature increases through the phase transition. Also, the correlations drop faster for next-nearest neighbors (triangles) than for nearest neighbors (squares). The value of $k T$ at which the orientations become essentially random is of the same order as the energy difference $\Delta \phi$ between the minima of the potential and the saddle points between them; for the case considered, $k T=0.09 \epsilon$ and $\Delta \phi=0.03 \epsilon$.

The specific heat data in Fig. 6 tend to the equipartition value of $\left(\frac{3}{2}\right) k$ as $T$ goes to zero. This is expected since each molecule has two translational and one rotational degree of freedom and, at sufficiently low temperatures, one expects the forces to be approximately harmonic.

The model gives a simple picture of the S to LS transition in fatty acid monolayers that is consistent with experimental results. At low temperatures, the anisotropy in the intermolecular potential prevents the molecules from rotating. As the temperature is increased, this constraint on molecular rotations becomes weaker until, at the phase transition, the molecules become effectively isotropic and pack in the hexagonal structure.

The quantitative discrepancy between experiment and simulation is not surprising considering the extreme simplicity of the model. The fact that the model system transition begins at lower temperature than the experimental transition is consistent with the approximation of nearly circular objects. Increasing $\alpha$ does increase the anisotropy in the potential, but this anisotropy is evaluated only along a line between centers for efficiency and simplicity. This limits the barrier to rotation, as evidenced by the immediate decrease in $C^{g}$ in Fig. 7. Further quantitative improvement may be achieved by extension from planar cross sections to cylindrical shapes of appropriate cross section. Nevertheless, the success of this model argues for the premise of Langmuir that monolayer packings are determined predominantly by the cross section of the component amphiphiles and offers a simple model for understanding the $\mathbf{S}$ and LS phases and the transition between them.

\section{ACKNOWLEDGMENTS}

We wish to thank C. H. Wang for useful discussions and D.R.S. acknowledges fellowship support from the University of Nebraska Foundation. Primary funding of this research is by ARO Grant No. DAAH04-93-G-0159.

${ }^{1}$ G. L. Gaines, Jr., Insoluble Monolayers at Liquid-Gas Interfaces (Interscience, New York, 1966).

${ }^{2}$ C. M. Knobler, Adv. Chem. Phys. 77, 397 (1990).

${ }^{3}$ H. M. McConnell, Annu. Rev. Phys. Chem. 42, 171 (1991).

${ }^{4}$ C. M. Knobler and R. C. Desai, Annu. Rev. Phys. Chem. 43, 207 (1992).

${ }^{5}$ I. R. Peterson, V. Brzezinski, and R. M. Kenn, Langmuir 8, 2995 (1992).

${ }^{6}$ A. M. Bibo, C. M. Knobler, and I. R. Peterson, J. Phys. Chem. 95, 5591 (1991).

${ }^{7}$ I. Langmuir, J. Am. Chem. Soc. 39, 1848 (1917).

${ }^{8}$ M. Lundquist, Ark. Kem. 17, 183 (1961); 21, 395 (1963); 23, (1965).

${ }^{9}$ C. J. Eckhardt, N. M. Peachey, D. R. Swanson, J. M. Takacs, M. A. Khan, X. Gong, J.-H. Kim, J. Wang, and R. A. Uphaus, Nature 362, 614 (1993).

${ }^{10}$ C. J. Eckhardt and D. R. Swanson, Chem. Phys. Lett. 194, 370 (1992).

${ }^{11}$ D. R. Swanson and C. J. Eckhardt, Langmuir 9, 22 (1992).

${ }^{12}$ D. K. Schwartz, M. L. Schlossman, and P. S. Pershan, J. Chem. Phys. 96, 2356 (1992).

${ }^{13}$ M. C. Shih, T. M. Bohanon, J. M. Mikrut, P. Zschack, and P. Dutta, J. Chem. Phys. 97, 4485 (1992).

${ }^{14}$ M. C. Shih, T. M. Bohanon, P. Zschack, J. M. Mikrut, and P. Dutta, Phys. Rev. A 45, 5734 (1992).

${ }^{15}$ S. Stallberg-Stenhagen and E. Stenhagen, Nature (London) 156, 239 (1945).

${ }^{16} \mathrm{~J}$. Doucet, I. Denicolo, and A. Craievich, J. Chem. Phys. 75, 1523 (1981).

${ }^{17}$ I. Denicolo, J. Doucet, and A. F. Craievich, J. Chem. Phys. 78, 1465 (1983).

${ }^{18}$ E. B. Sirota, H. E. King, Jr., D. M. Singer, and H. H. Shao, J. Chem. Phys. 98, 5809 (1993).

${ }^{19}$ R. M. J. Cotterill, Biochim. Biophys. Acta 433, 264 (1976).

${ }^{20} \mathrm{~S}$. Toxvaerd, J. Chem. Phys. 67, 2056 (1977).

${ }^{21}$ For example, V. M. Kaganer, M. A. Osipov, and I. R. Peterson, J. Chem. Phys. 98, 3512 (1993); S. Karaborni and S. Toxvaerd, ibid. 97, 5876 (1992); M. Scheringer, R. Hilfer, and K. Binder, ibid. 96, 2269 (1992); D. Kramer, A. Ben-Shaul, Z.-Y. Chen, and W. M. Gelbert, ibid. 96, 2236 (1992); Z.-G. Wang, J. Phys. 51, 1431 (1990).

${ }^{22}$ For example, N. Collazo, S. Shin, and S. A. Rice, J. Chem. Phys. 96, 4735 (1992); S. Shin, N. Collazo, and S. A. Rice, ibid. 98, 3469 (1993); J. P. Bareman and M. L. Klein, J. Phys. Chem. 94, 5202 (1990).

${ }^{23}$ T. Kihara, Intermolecular Forces (Wiley, Chichester, 1978).

${ }^{24}$ R. A. Sack, Mol. Phys. 2, 8 (1959).

${ }^{25}$ I. R. McDonald, Mol. Phys. 23, 41 (1972). 